\title{
The Three-Dimensional Velocity Distribution of Wide Gap Taylor-Couette Flow Modelled by CFD
}

\author{
David Shina Adebayo and Aldo Rona \\ Department of Engineering, University of Leicester, University Road, Leicester LE1 7RH, UK \\ Correspondence should be addressed to Aldo Rona; ar45@le.ac.uk
}

Received 9 October 2015; Revised 1 February 2016; Accepted 2 February 2016

Academic Editor: Ryoichi Samuel Amano

Copyright ( 2016 D. S. Adebayo and A. Rona. This is an open access article distributed under the Creative Commons Attribution License, which permits unrestricted use, distribution, and reproduction in any medium, provided the original work is properly cited.

\begin{abstract}
A numerical investigation is conducted for the flow between two concentric cylinders with a wide gap, relevant to bearing chamber applications. This wide gap configuration has received comparatively less attention than narrow gap journal bearing type geometries. The flow in the gap between an inner rotating cylinder and an outer stationary cylinder has been modelled as an incompressible flow using an implicit finite volume RANS scheme with the realisable $k-\varepsilon$ model. The model flow is above the critical Taylor number at which axisymmetric counterrotating Taylor vortices are formed. The tangential velocity profiles at all axial locations are different from typical journal bearing applications, where the velocity profiles are quasilinear. The predicted results led to two significant findings of impact in rotating machinery operations. Firstly, the axial variation of the tangential velocity gradient induces an axially varying shear stress, resulting in local bands of enhanced work input to the working fluid. This is likely to cause unwanted heat transfer on the surface in high torque turbomachinery applications. Secondly, the radial inflow at the axial end-wall boundaries is likely to promote the transport of debris to the junction between the end-collar and the rotating cylinder, causing the build-up of fouling in the seal.
\end{abstract}

\section{Introduction}

The understanding of the flow in the gap between concentric independently rotating cylinders is both of scientific and of practical interest for many engineering applications in rotating machinery. Specific examples include the lubricating flow between rotating shafts of turbopumps in rocket engines and of multispool turbofan engines and in the bearing housing of high [1] and low [2] bypass aircraft engines. Other areas of application are found in the bearing chambers of internal combustion aero-engines, rotating tube in tube heat exchangers, and the submerged pumps for water wells.

Lubrication is very important in turbomachineries where the inner cylinder (shaft) rotates and the outer cylinder (journal bearing) is stationary. In this application, the clearance is typically small enough, the lubricant is viscous enough, and the speeds are slow enough so that the flow is laminar. The flow is eccentric because the radial loading on the shaft reduces the bearing clearance on one side. With a properly designed bearing, the shaft, while turning, will not contact the bearing because the viscous shear force between the shaft and the lubricant carries the lubricant into this space. At high shaft speeds and high shaft loads, the laminar flow becomes first axially nonuniform and then nonaxisymmetric [3-9]. This is because the forces arising from viscosity are insufficient to overcome those associated with the fluid inertia. This transition increases the shaft torque significantly so that ball and or roller bearings are used in place of a journal bearing at these higher rotational speeds.

Many experimental investigations and numerical simulations have been conducted to understand the complexities of this flow. This activity dates back to 1888 and 1890, when Mallock [3, 4] and Couette [5] conducted independent experiments using concentric rotating cylinders. More recently, Liao et al. [8] conducted numerical simulations that reproduced three regimes of the Couette-Taylor system, namely, the steady circular Couette flow, the steady axisymmetric Taylor vortex flow, and the periodic spiral vortex flow. They validated their computational results using the experimental observations of Andereck et al. [9]. They concluded that 


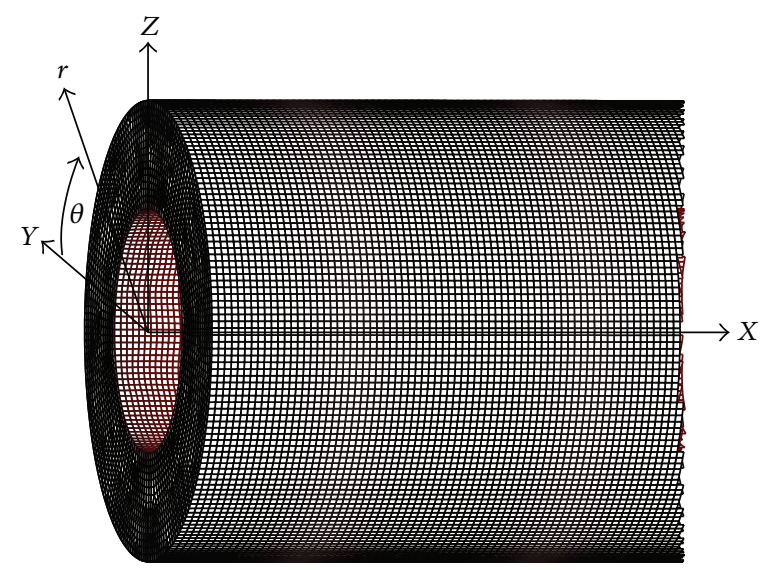

(a)

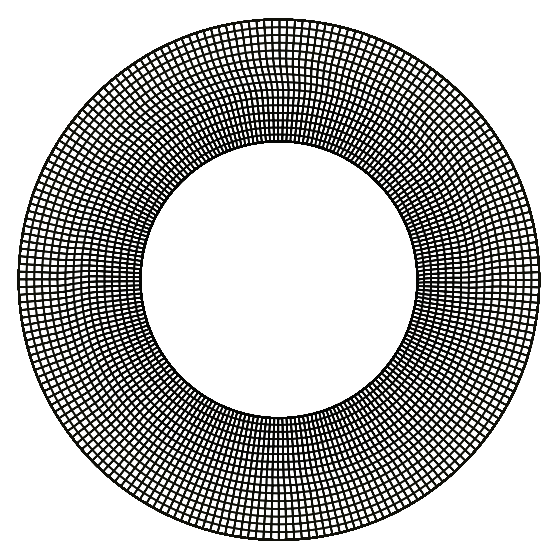

(b)

FIGURE 1: (a) Tetrahedral computational mesh structure and (b) computational mesh detail at the end-wall. One mesh point every 10 has been plotted radially, for clarity.

this system exhibits a rich diversity of steady and chaotic flow patterns that are complex in nature and may arise as a result of small perturbations. These characteristics are typical expressions of hydrodynamic instabilities in the flow. Czarny et al. [10] performed a direct numerical simulation, using a three-dimensional spectral method, of a small axial length to diameter ratio annular flow driven by counterrotating cylinders. The numerical model predicted two different flow regimes, wavy vortices and interpenetrating spirals.

The flow enclosed between rotating coaxial cylinders is often characterised with respect to the Taylor number, Ta, which expresses in nondimensional form the importance of the centripetal acceleration in a rotating flow relative to the viscous forces. In this study, where only the inner cylinder is rotating, the Taylor number Ta is defined as

$$
\mathrm{Ta}=\frac{2 \eta^{2} d^{4}}{1-\eta^{2}}\left(\frac{\Omega}{v}\right)^{2}
$$

where $\eta=R_{i} / R_{o}$ is the radius ratio, $R_{i}$ and $R_{o}$ are the radii of the inner and the outer cylinders respectively, $d=R_{o}-R_{i}$ is the gap width, $\Omega$ is the rotational speed of the inner cylinder, and $\nu$ is the fluid kinematic viscosity.

Many aspects of the flow developing between coaxial rotating cylinders are yet to be fully detailed. One of the advantages of $3 \mathrm{D}$ simulations over traditional experiments is the ability to investigate the salient features of the flow across the entire annulus on meridional, axial, and cascade planes. Whilst a 3D model is more demanding both in terms of its development time and of the computational resources, it has the potential to resolve the time-averaged three-dimensional motion of the localised flow disturbances induced by the rotation of the inner cylinder.

Adebayo and Rona [11, 12] measured by PIV the inplane velocity between rotating cylinders at wide gap. These measurements were limited to the meridional plane where the PIV gave direct measurements of the in-plane velocity. Computational fluid dynamic (CFD) can overcome this limitation by estimating the full $3 \mathrm{D}$ velocity field as part of the flow field solution. The CFD model therefore enables quantifying and qualifying the important flow fields beyond the current limitations of conventional nonintrusive opticsbased measurement techniques.

In this study, CFD is used to predict the flow pattern and examine in detail the velocity distributions both in the meridional and in the axial planes in a moderately wide gap setup. The accuracy of the predicted result is validated by comparing the velocity profiles from the CFD simulations to the PIV measurements by Adebayo and Rona [11, 12] in the meridional plane. Conclusions are drawn on the significant implication of these findings for high torque turbomachinery applications.

\section{Computation Domain and Flow Conditions}

2.1. Geometry. A three-dimensional (3D) numerical model is used to examine the velocity field flow in more than one plane. The model geometry is defined with respect to the coordinates shown in Figure 1. The cylinders are coaxial with the axis coinciding with the $X$-direction of the cylindrical reference system $(r, \theta, X)$. Two different coaxial assemblies, summarised in Table 1, are considered to allow a parametric study of the flow pattern in the annular region of the coaxial cylinders. The rotating speed $\Omega$ of the inner cylinder is held constant at $52.36 \mathrm{rad} / \mathrm{s}$ in all test cases. The geometries modelled in this study were created using commercial CFD software GAMBIT 2.4.6.

2.2. Boundary Conditions. The internal flow between the two rotating cylinders is modelled as fully enclosed using the commercial CFD software ANSYS Fluent [13]. At the start of the computation, zero flow conditions are imposed throughout the computational domain. The axis of the cylinders is horizontal and the vertical extent of computational domain is limited to $0.114 \mathrm{~m}$. Therefore, gravitational acceleration effects are not modelled. No-slip stationary adiabatic wall boundary conditions are applied at the left and the right 
TABLE 1: Specifications for the computational parameters.

\begin{tabular}{lcc}
\hline Description & Test case 1 & Test case 2 \\
\hline Inner cylinder length $(\mathrm{m})$ & 0.5 & 0.5 \\
Outer cylinder length $(\mathrm{m})$ & 0.5 & 0.5 \\
Inner cylinder outer diameter $(\mathrm{m})$ & 0.05 & 0.05 \\
Outer cylinder inner diameter $(\mathrm{m})$ & 0.094 & 0.114 \\
Gap width $d(\mathrm{~m})$ & 0.022 & 0.032 \\
Aspect ratio $\Gamma=L / d$ & 11.36 & 7.81 \\
Radius ratio $\eta=R_{i} / R_{o}$ & 0.53 & 0.44 \\
Clearance ratio $d / R_{i}$ & 0.88 & 1.28 \\
Reynolds number $\mathrm{Re}$ & $1.97 \times 10^{3}$ & $2.88 \times 10^{3}$ \\
Taylor number Ta & $2.35 \times 10^{6}$ & $6.47 \times 10^{6}$ \\
\hline
\end{tabular}

end-walls, as well as the outer cylindrical surface at $R_{o}$. The inner cylinder surface is modelled as a rotating adiabatic noslip wall. These boundaries fully delimit the computational domain and define a closed system. An adequate match of the acceleration profile between experiment [11, 12] and computation was found to be important and the simulations in this work were carried out with a sudden start of the inner cylinder at the specified angular speed, modelling experiments in which the target rotational speed of the inner cylinder is reached within one second $[14,15]$.

The closed type system being modelled enables some flexibility in the definition of the turbulence quantities at the start of the computation. Unlike an open system with a computational inflow boundary, which affects the solution at all times, the turbulence quantities in a closed system are updated by the flow solver and are therefore self-determined in the converged solution. The turbulence level at the start of the computation was specified in terms of the turbulence intensity and the hydraulic diameter. Estimations of turbulence intensity TI, turbulent kinetic energy $k$, and turbulent kinetic energy dissipation rate $\varepsilon$ were calculated for all the CFD simulations using empirical correlations for pipe flows [13]. Specifically,

$$
\begin{aligned}
\mathrm{TI} & =0.16 \mathrm{Re}_{H}^{-0.125}, \\
k & =1.5 U_{\mathrm{avg}}^{2} \mathrm{TI}^{2}, \\
\varepsilon & =C_{\mu}^{0.75} k^{1.5} l^{-1},
\end{aligned}
$$

where $\operatorname{Re}_{H}$ is the Reynolds number based on the hydraulic diameter $D_{H}=2 d$ of the cylindrical assembly, $U_{\text {avg }}$ is the bulk velocity, the constant $C_{\mu}=0.09$, and $l=0.07 D_{H}$.

The turbulence intensity, turbulent kinetic energy $k$, and turbulent kinetic energy dissipation rate $\varepsilon$ levels are $6 \%$, $0.0093 \mathrm{~m}^{2} / \mathrm{s}^{2}$, and $0.0478 \mathrm{~m}^{2} / \mathrm{s}^{3}$, respectively, for the test case $\Gamma=11.36$, and $5 \%, 0.0064 \mathrm{~m}^{2} / \mathrm{s}^{2}$, and $0.0189 \mathrm{~m}^{2} / \mathrm{s}^{3}$, respectively, for the test case $\Gamma=7.81$. These are indicative of a turbulent flow in the annular region between the cylinders and are above typical values of $0.2 \%$ to $0.3 \%$ that are found in the test section of well-designed wind tunnels.

\section{Numerical Model}

3.1. Computational Scheme. In this study, all the CFD simulations assume an isothermal, viscous, and incompressible (constant density) fluid. The incompressible Reynolds Averaged Navier Stokes (RANS) equations for the conservation of mass and momentum $[16,17]$ govern this flow. The governing equations are discretized by a finite volume approach and the pressure based segregated solver by Chorin [18] is used for their numerical integration. At each finite volume face, the values of the integrand are required for the convection terms of the turbulence closure model. This is determined by interpolation from the cell-averaged flow state and this is accomplished by using a second-order upwind scheme [17].

In this study, two turbulence models were evaluated for closing the RANS equations: the realisable $k-\varepsilon$ model developed by Shih et al. [19] and the Reynolds stress model (RSM) by Launder et al. [20]. These models were used to run identical cases for each of the two computational geometries. The results show that the realisable $k-\varepsilon$ model predicts higher radial velocity maxima $(0.2 \%)$ and lower radial velocity minima $(0.1 \%)$ than the RSM model. This indicates that stronger Taylor vortices are predicted with the realisable $k-\varepsilon$ model. Apart from these quantitative differences between the predictions from the two models, the RSM took at least $50 \%$ more CPU time to complete the simulations for all test cases. Based on the result from this preliminary test, the realizable $k-\varepsilon$ turbulence model was chosen for computing all the CFD solutions in this study. The selection of the realizable $k-\varepsilon$ model for the RANS study is also supported by past experience with CFD. The realizable $k-\varepsilon$ model provides superior performance than the standard $k-\varepsilon$ model for flows involving rotation, boundary layers under strong adverse pressure gradients, separation, and recirculation [13] like in this study. The realizable $k-\varepsilon$ model has also shown substantial improvements over the standard $k-\varepsilon$ model where the flow features include strong streamline curvature, vortices, and rotation.

3.2. Domain Discretisation. The computational domains were meshed using the commercial CFD mesh generator Gambit 2.4.6. The unstructured tetrahedral mesh of intermediate mesh density (type 2) used for test case 1 is shown in Figure 1. The mesh quality of the two test cases was assessed by evaluating the cell skewness. The maximum cell skewness for the test cases was found to be within the range 0.500.7 , which suggests that all the meshes in this work are of good quality [13]. The mesh was clustered around the inner cylinder surface by the size function tool in GAMBIT 2.4.6. A constant starting cell size of $1.0 \mathrm{~mm}$ was used for all the test cases. This value was increased by $50 \%$ and $40 \%$ for growth rate and maximum cell size, respectively, for the test cases $\Gamma=11.36$ and $\Gamma=7.81$.

A grid independence test was conducted to assess the dependence of the results upon the level of the spatial discretisation. Three progressively finer meshes were evaluated for the two test cases in this study. For the test case $\Gamma=11.36$, 4.2 million, 6.1 million, and 7.9 million cells were used. For the test case $\Gamma=7.81,4.9$ million, 6.9 million, and 7.9 million 


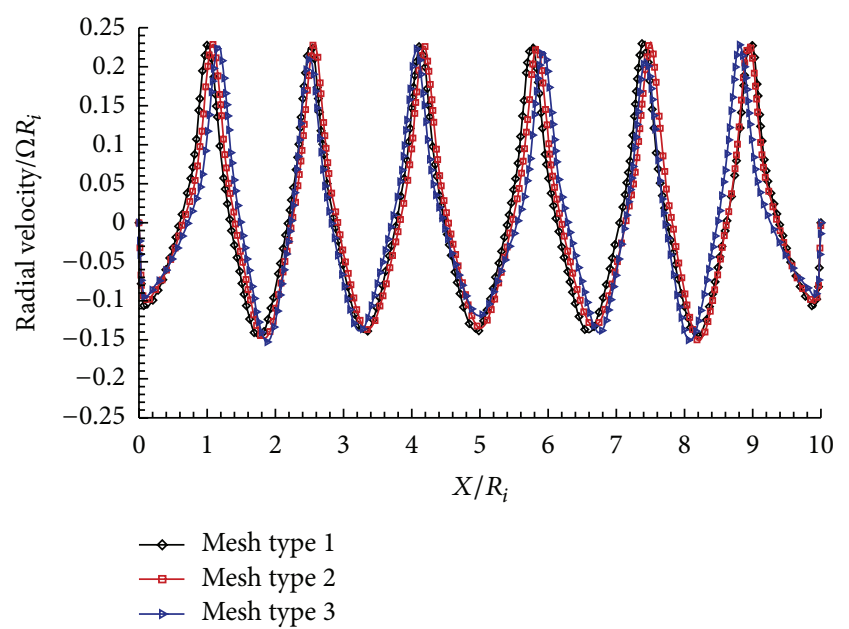

(a)

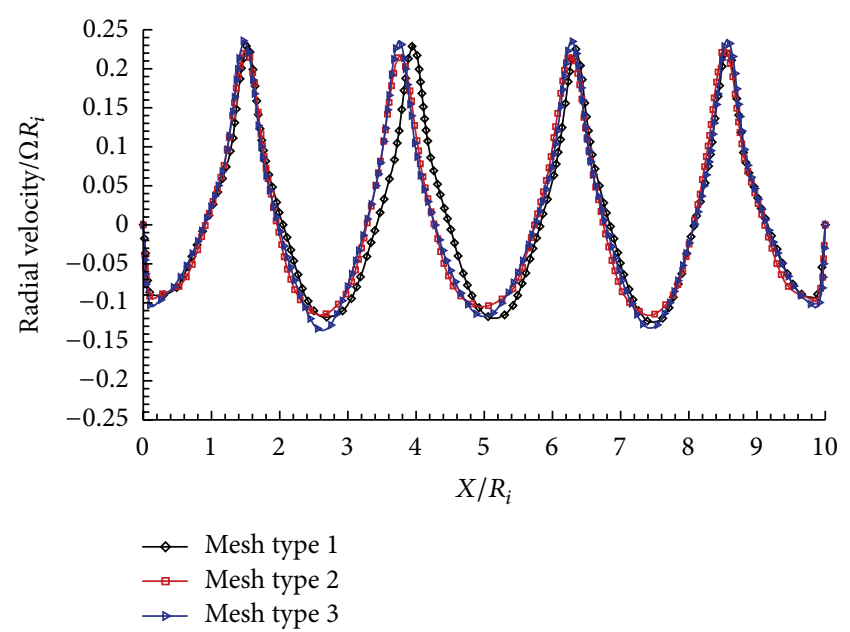

(b)

FIGURE 2: Radial velocity profiles at $r=R_{i}+0.5 d$ for three different levels of computational mesh refinement for test cases (a) $\Gamma=11.36$ and (b) $\Gamma=7.81$.

cells were used. These meshes are referred to as type 1, type 2 , and type 3 in the progressive mesh size order in Figure 2. The predictions of the total number of vortices and of the axial distribution of static pressure, axial velocity, and radial velocity were used as monitoring parameters to establish the grid independence of the predictions.

Figures 2(a) and 2(b) show, respectively, the CFD predictions of the radial velocity profiles extracted at the gap midspan of the lower channel $(\theta=-\pi / 2)$ in the meridional plane for the two test cases using the three different levels of computational mesh refinement. The results show that the radial velocity profiles have the same trends, which indicate that the different levels of computational mesh refinement have little impact on the axial spacing of the Taylor vortices, as shown by the regularly spaced maxima and minima in Figures 2(a) and 2(b).

Predictions obtained using mesh types 1 to 3 display an appreciable overlap in radial velocity over the entire range $0 \leq X / R_{i} \leq 10$. This indicates that the numerical scheme is predicting the same Taylor vortex pattern at all the three levels of mesh refinement and that any further increase in grid density may not noticeably improve the accuracy of the RANS predictions.

The percentage differences in the radial velocity between mesh type 3 and the other two mesh types were calculated. The results show that the percentage differences between the mesh types were all less than 5\% for all the flow variables, while the number of the vortices for the two test cases remains unchanged. As a result, the computational mesh type 2 was chosen for the two test cases in this study as it provides sufficiently grid independent predictions, given the available computational resources.

\section{Validation of the CFD Results}

In the experiment by Adebayo and Rona [11, 12], the inplane velocity was surveyed by PIV on the meridional plane.
The comparison of the CFD predictions with these reference measurements shows that there is agreement between the two results. For instance, Figure 3 shows the axial and radial velocity profiles from the CFD simulations and the PIV experiments for the test case $\Gamma=11.36$. The velocity is normalised with respect to the inner cylinder surface speed $\Omega R_{i}$ and lengths are normalised by the inner cylinder radius $R_{i}$. The velocity profiles were extracted and plotted at constant radial positions $r=R_{i}+0.125 d$ for the axial velocity and at $r=R_{i}+0.5 d$ for the radial velocity, along the axial direction on the lower $(\theta=-\pi / 2)$ channel of the annulus. The PIV error bands delimited by the plain dashed lines (without symbols) are included in the profiles of Figures 3(a) and 3(b) to document the experimental uncertainty.

In Figures 3(a) and 3(b), the velocity profiles from both CFD and PIV at the radial positions $r=R_{i}+0.125 d$ and $r=R_{i}+0.5 d$, respectively, show that the flow is almost centrally symmetric about the cylinder length midspan at $X / R_{i}=5$ and the magnitude of the corresponding velocity minima and maxima is almost the same. Although there is a little variation between the PIV and the CFD velocity profiles near the right end-wall in Figures 3(a) and 3(b), the CFD profiles are still within the PIV measurement uncertainty band. The little difference observed between the PIV and the CFD profiles in Figures 3(a) and 3(b) at the end-wall boundaries is attributed to the approximate alignment of the end-wall boundaries orthogonal to the cylinder axis in the experimental setup.

Overall, the spatial variation of the in-plane velocities observed by PIV is substantially reproduced by the CFD simulations. This indicates that the time averaged flow dynamics is correctly modelled.

\section{Results and Discussions}

5.1. Overall Flow Resistance to Rotation. The tangential velocity difference between the inner and the outer cylindrical 


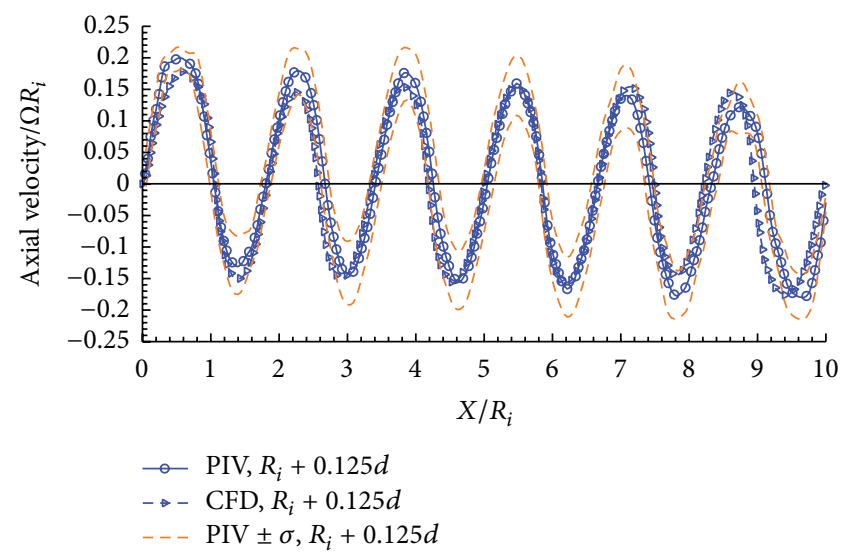

(a)

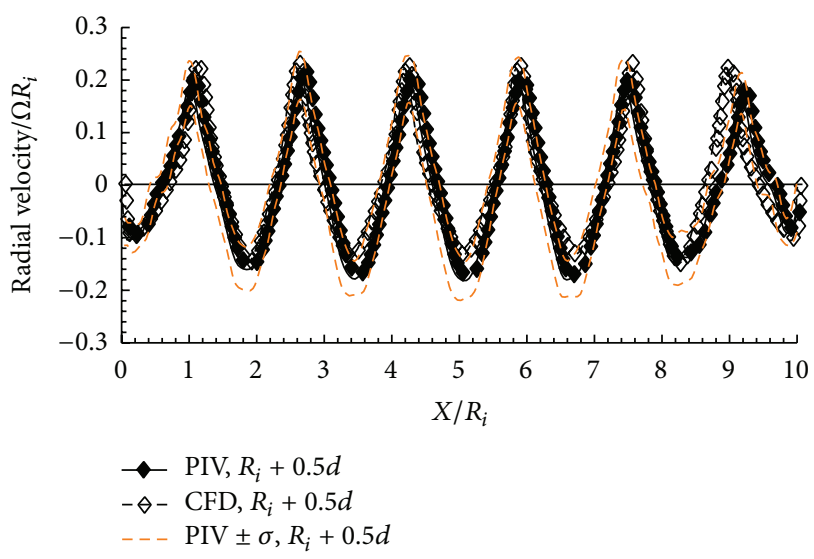

(b)

FIgURE 3: Normalised (a) axial and (b) radial velocity profiles from PIV and CFD at the constant radial positions $r=R_{i}+0.125 d$ and $r=R_{i}+0.5 d$ on the meridional plane $\theta=-\pi / 2$, with the PIV error band. $\Gamma=11.36$.

walls shears the fluid in the annular gap to resist the rotation of the inner cylinder. This resistance manifests itself as a torque, which is an integral quantity, dependent on the flow pattern. The torque predicted by CFD from integrating the surface shear stress on the inner cylinder is $2.09 \times 10^{-5} \mathrm{Nm}$ and $2.06 \times 10^{-5} \mathrm{Nm}$, respectively, for the test cases $\Gamma=11.36$ and $\Gamma=7.81$. Comparative values of torque $T$ were obtained from the empirical correlation of Wendt [21] reported in Lathrop et al. [22]

$$
\frac{T}{\rho v^{2} L}=1.45 \frac{\eta^{3 / 2}}{1-\eta^{7 / 4}} \operatorname{Re}^{1.5}
$$

where $\rho$ is the air density, $L=0.25 \mathrm{~m}$ is the axial length of the cylinders, and $\operatorname{Re}=\sqrt{\operatorname{Ta}(1-\eta)^{-1}(1+\eta) / 2}$ is the flow Reynolds number. At the test conditions of Table 1 , the torque estimates are $1.22 \times 10^{-5} \mathrm{Nm}$ and $1.16 \times 10^{-5} \mathrm{Nm}$, respectively, for the test cases $\Gamma=11.36$ and $\Gamma=7.81$. As the empirical correlation does not include any information about the staging between different Taylor vortex regimes, the match in the order of magnitude between the predicted torque from the empirical correlation and CFD may be deemed satisfactory. Given the very small magnitude of these torque values, a direct measurement of this variable was not attempted in Adebayo and Rona $[11,12]$ due to the dominant role of the torque contribution from friction in the roller bearings that supported the rotation of the inner cylinder.

5.2. Flow Pattern in the Meridional Plane. Figures 4(a) and 4(b) show, respectively, the CFD predictions of the velocity vectors in the meridional plane for the configurations $\Gamma=$ 11.36 and $\Gamma=7.81$. The velocity vectors are normalised by the inner cylinder tangential speed $\Omega R_{i}$. An axisymmetric flow pattern of pairs of counterrotating vortices is shown in both Figures 4(a) and 4(b). This pattern repeats along the axial shaft with consecutive cells moving the flow in the same direction at their meeting point. Along the axial direction of the computational domain, which is $10 R_{i}$, six pairs of vortices are predicted for the test case $\Gamma=11.36$, while four pairs of vortices are predicted for the test case $\Gamma=7.81$. These are symmetrical about the midpoint along the axial direction. Therefore, to illustrate the flow pattern more clearly, only the vortices to the left of the point of symmetry are shown in Figure 4. The reduction in the number of the vortices from six pairs to four pairs is one of the effects of the change in the gap width $d$ and an indication that the number of vortices is a function of the aspect and radius ratios, in agreement with the experimental investigation by Adebayo and Rona [11, 12].

In the CFD simulation, the no-slip boundary conditions imposed at the end-walls generate a boundary layer flow, with a peculiar discontinuity in velocity at the junctions between the rotating inner cylinder and the stationary end-walls. The discontinuity of the boundary conditions at the meeting point of the inner cylinder and the end-walls generates a weaker circulation cell adjacent to these end-walls compared to the more inbound cells, as can be observed in Figure 4.

As the inner cylinder rotates, the radial velocity induced by the vortices, as a result of a higher centrifugal force experienced by the fluid particles near the wall of the inner cylinder, convects the fluid with high tangential momentum near the rotating inner cylinder radially outward, in the outflow regions between two adjacent paired vortices. Symmetrically, low speed fluid from near the stationary outer cylinder is convected radially inward in the inflow (upwell at $\theta=-\pi / 2$ ) regions between two adjacent pairs of vortices. The redistribution of the angular momentum of the fluid across the annulus affects the inward and the outward flow velocity distribution. This is responsible for the stronger radial outflow than the radial inflow between the vortices observed in Figure 4.

At the meeting point of two adjacent vortices, there are significant flow mixing and exchange of momentum at the outflow region, with each vortex adding to the mixing region at the centre of a vortex pair, close to the inner cylinder, and then receiving fluid from this mixing region, close to the outer cylinder. A similar mixing process occurs at the inflow region, between neighbouring vortex pairs. 


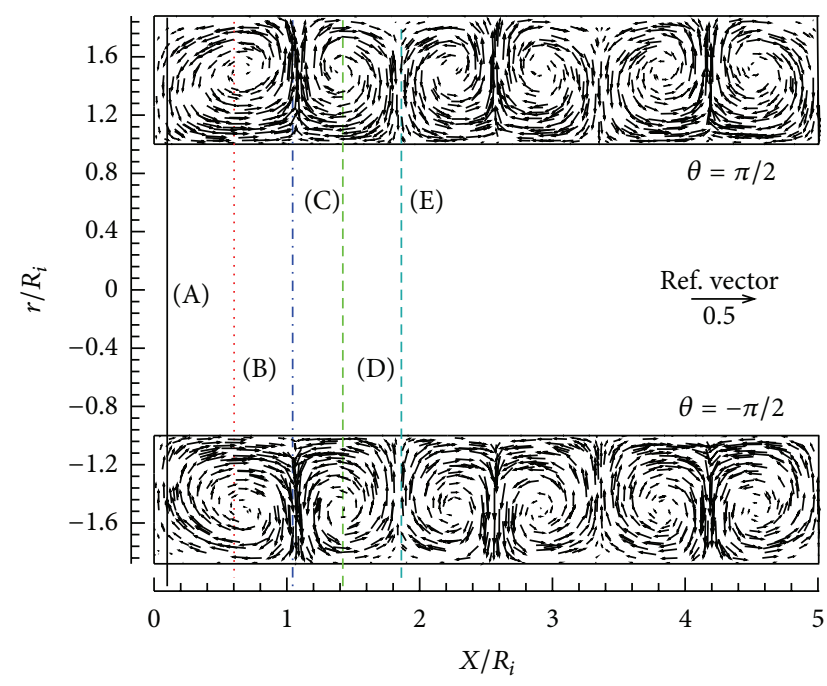

(a)

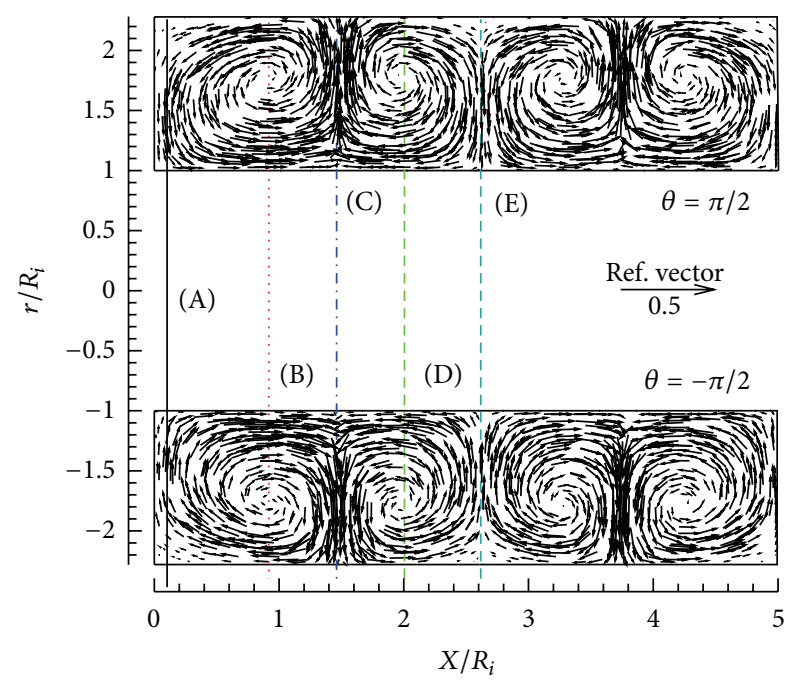

(b)

Figure 4: Normalised velocity vectors in the meridional plane of the annulus for test cases (a) $\Gamma=11.36$ and (b) $\Gamma=7.81$. The reference velocity vector magnitude is $0.5 \Omega R_{i}$.

In Figure 4, the vortex centres are shifted toward the outer cylinder, due primarily to the imbalance between the centrifugal force due to the rotation of the inner cylinder and the pressure gradient due to the stationary outer cylinder wall, as the centrifugal force pulls the fluid away from the rotating inner cylinder.

5.3. Flow Pattern in the Axial Plane. Figures 5(a)-5(f) show the velocity vector maps of different flow patterns at various axial locations projected on the axial plane for the test cases $\Gamma=11.36$ and $\Gamma=7.81$. The analysis of the flow in the axial plane complements the PIV results obtained in the meridional plane reported by Adebayo and Rona $[11,12]$ to achieve a clearer understanding of the physics of the fluid motion in the annular gap between the cylinders as the gap width changes. Various cross sections have been sliced normal to the axis of the coaxial cylinders of Figure 1 in order to analyse the flow variables in the axial planes. In all, six different flow patterns (including the flow pattern at the endwalls) are identified within the computational domain. Four out of the six patterns recur in the axial direction with the spatial period of one Taylor vortex pair. The sliced positions are labelled alphabetically (A-E) in Figures 4(a) and 4(b) for the test cases $\Gamma=11.36$ and $\Gamma=7.81$, respectively, for easy identification. The location of slice $\mathrm{F}$, which is not shown in these figures, is close to the right end-wall at $X / R_{i}=9.95$. These sections define the axial planes where the flow pattern is investigated and corresponds to axial positions (A) $X / R_{i}=$ 0.05 , (B) $X / R_{i}=0.65$, and (C) $X / R_{i}=1.07$, in Figure 4(a), and (D) $X / R_{i}=1.97$ and (E) $X / R_{i}=2.62$, in Figure 4(b).

The velocity vectors maps at $X / R_{i}=1.41, X / R_{i}=1.84$, and $X / R_{i}=9.95$ for the test case $\Gamma=11.36$ give similar patterns as the velocity vector maps in Figures 5(d)-5(f) for test case $\Gamma=7.81$ and are therefore not shown. Similarly, velocity vectors maps at $X / R_{i}=0.05, X / R_{i}=0.92$, and
$X / R_{i}=1.47$ for the test case $\Gamma=7.81$ are not shown because they are similar to the velocity vector maps in Figures 5(a)5(c) for test case $\Gamma=11.36$. In Figure 5, the reference vector is shown on each velocity map so that the magnitude of the vectors can be appreciated.

Figures 5(a) and 5(f) show, respectively, the normalised velocity vector maps near the end-walls at $X / R_{i}=0.05$ and $X / R_{i}=9.95$ for the test cases $\Gamma=11.36$ and $\Gamma=7.81$ and enable understanding the effects of the end-walls on the axial plane flow. The magnitudes of the velocity vectors at these locations are relatively small compared with other locations in the central region of the computational domain. This is primarily because computations are performed with a stationary end-wall.

Figures 5(b) and 5(d) show the normalised velocity vectors at $X / R_{i}=0.65$ at $\Gamma=11.36$ and at $X / R_{i}=1.97$ at $\Gamma=7.81$. These locations correspond to the centres of a clockwise vortex and of an anticlockwise vortex, respectively, in Figures 4(a) and 4(b) based on the flow visualisations of the lower channel at $\theta=-\pi / 2$ of Figures $4(\mathrm{a})$ and $4(\mathrm{~b})$. The flow patterns of Figures 5(b) and 5(d) for the test cases $\Gamma=11.36$ and $\Gamma=7.81$ are similar in terms of the velocity vector magnitude, despite the difference in the gap width, while, in the meridional plane of Figure 4, the axial velocity components at (B) and (D) are opposite to one another. The axial location of Figure 5(b) corresponds to an axial location of almost zero radial velocity in Figure 3(b) and to an axial velocity maximum in Figure 3(a). Similarly, the axial location of Figure 5(d) corresponds to an axial location of almost zero radial velocity in Figure 2(b).

In Figure 5(c), the axial plane $X / R_{i}=1.07$ corresponds to the outward flow region in Figure 4(a). The flow pattern at this location shows that the magnitude of the velocity vectors is higher compared with the magnitude of the velocity 

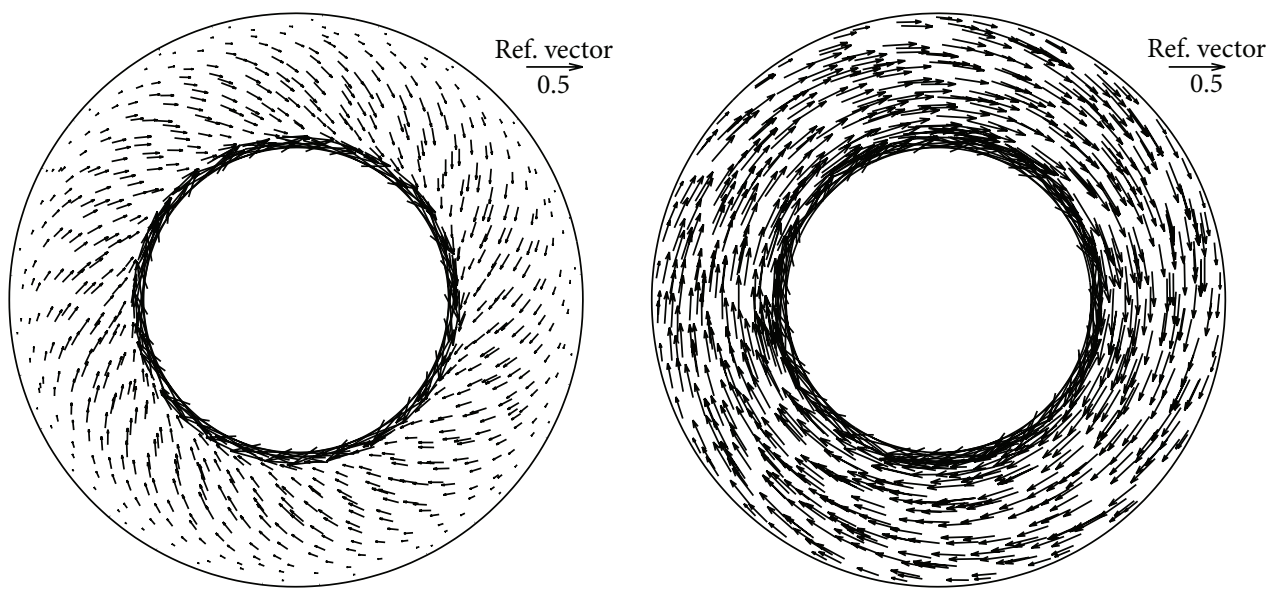

(a) $X / R_{i}=0.05, \Gamma=11.36$

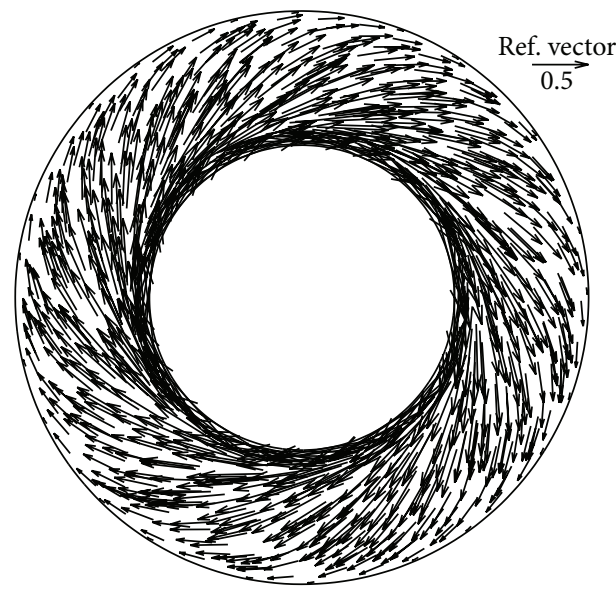

(b) $X / R_{i}=0.65, \Gamma=11.36$

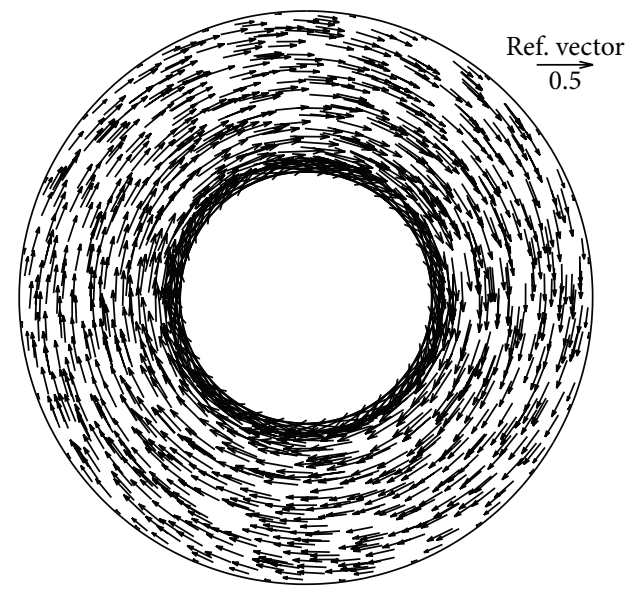

(c) $X / R_{i}=1.07, \Gamma=11.36$

(d) $X / R_{i}=1.97, \Gamma=7.81$
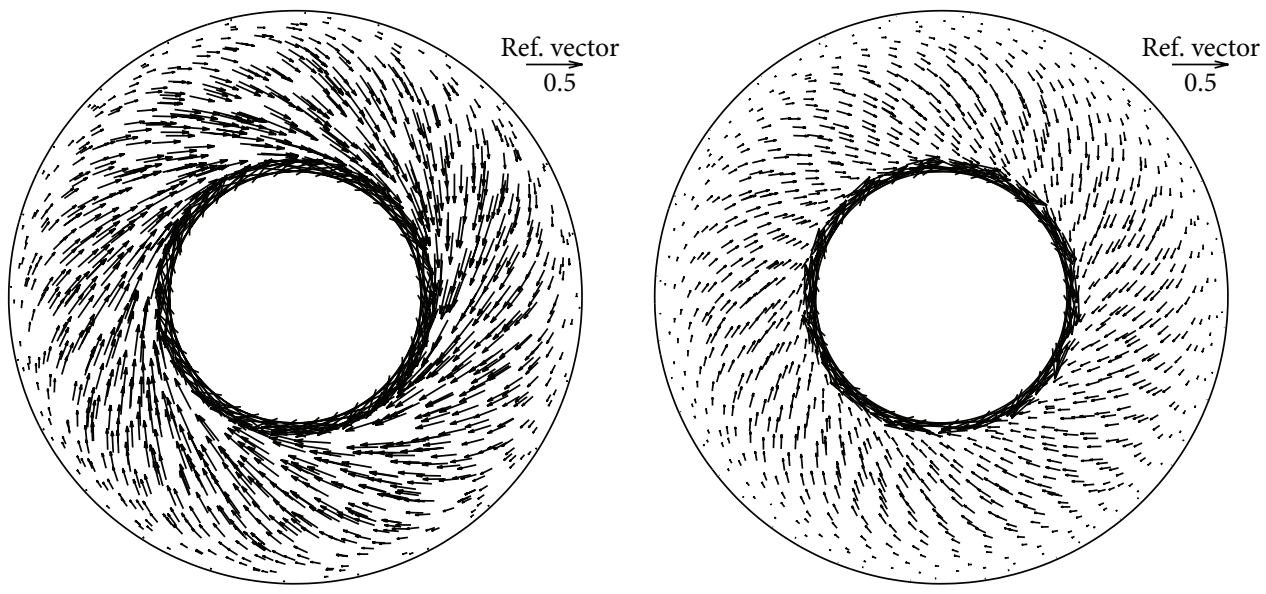

(e) $X / R_{i}=2.62, \Gamma=7.81$

(f) $X / R_{i}=9.95, \Gamma=7.81$

FIGURE 5: Velocity vectors in the axial plane normalised by $\Omega R_{i}$.

vectors at the other locations in Figure 5. This position is where high radial momentum fluid is being carried outwardly by the Taylor vortices toward the stationary outer cylinder, as evidenced by the direction of the velocity vector pattern. This position corresponds to an axial velocity zero crossing in Figure 3(a) and to a radial velocity maximum in Figure 3(b).
Figure 5(e) shows the normalised velocity vectors at $X / R_{i}=2.62$ for the test case $\Gamma=7.81$. This location corresponds to an inflow region in Figure 4(b) for the test case $\Gamma=7.81$. At this location, low tangential momentum fluid is being carried inwardly by the radial velocity induced by the Taylor vortices from the stationary outer cylinder toward 


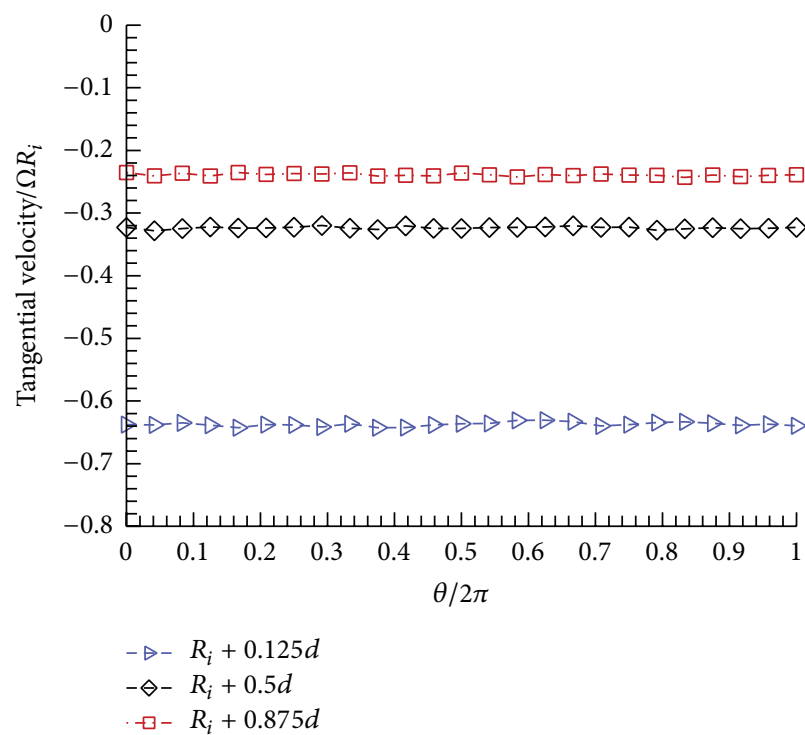

(a)

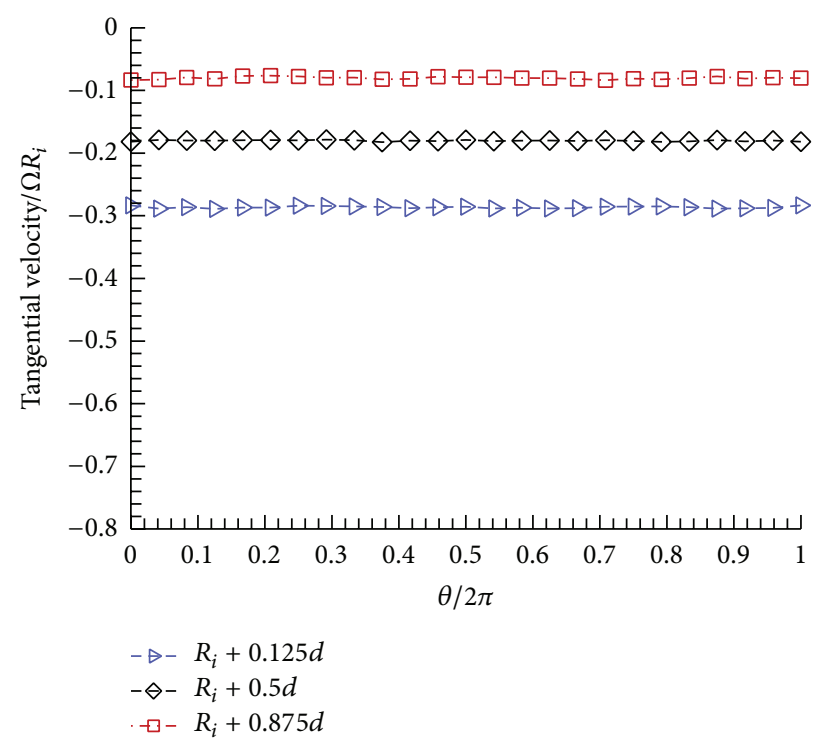

(b)

FIGURE 6: Azimuthal profiles of normalised tangential velocity at different radial heights on selected axial planes for the test cases (a) $\Gamma=11.36$ and (b) $\Gamma=7.81$.

the rotating inner cylinder, as evidenced by the direction of the velocity vector pattern. This position corresponds to a position of radial velocity minimum in Figure 2(b). Comparing the velocity vectors at this position to the velocity vectors in Figure 5(c), it is evinced that the tangential momentum at the inflow regions is lower than that at the outflow regions.

Figures 5(a)-5(f) show that the velocity vectors near the inner cylinder display a higher tangential velocity magnitude than the vectors near the outer cylinder. The tangential velocity at the wall of the outer stationary cylinder tends to zero, primarily to satisfy the no-slip condition imposed on the wall of the outer cylinder. These velocity vector maps indicate that the tangential velocity is the dominant in-plane velocity component, as the radial velocity is relatively weak.

5.4. Velocity Profiles in the Axial Plane. The azimuthal distribution of the tangential velocity at the axial location $x / R_{i}=$ 1.07 is shown in Figure 6(a). This is to examine the axial symmetry of the flow for the test case $\Gamma=11.36$ at constant radial positions $\left(r-R_{i}\right) / d$ of $0.125,0.5$, and 0.875 .

Figure 6 shows that at all three radial positions the tangential velocity is essentially constant and nearly independent from the azimuthal coordinate $\theta$. This indicates that the flow is substantially axisymmetric and that does not manifest any appreciable waviness of the Taylor vortices in the azimuthal direction. Similar results are presented in Figure 6(b) for the test case $\Gamma=7.81$ at the axial location $x / R_{i}=2.62$.

The radial velocity profiles at the axial locations (B) to (E) of Figure 4 were extracted for the test cases $\Gamma=11.36$ and $\Gamma=$ 7.81. The profiles in the axial plane are axisymmetric, as such the profiles at different angles $\theta$ around the annulus coincide. These profiles enable assessing the radial dependence of the flow variables more readily than the meridional and cascade plane plots. The profiles, therefore, can be used to provide information about the radial velocity distributions within the annular space of bearing chambers and for further clarifying the trends shown by the velocity vector maps in Figure 5.

Figures 7(a) and 7(b) show, respectively, the radial profiles of tangential velocity at a single axial location extracted from axial planes like (C) in Figures 4(a) and 4(b), along the full length of the cylinder. These planes are the centre of the outward flow regions (downwells) for the test cases $\Gamma=$ 11.36 and $\Gamma=7.81$. The predicted flow tangential velocity, normalised by the inner cylinder tangential rotational speed $\Omega R_{i}$, is plotted as clockwise negative in Figures 6 and 7. This makes the sign of the tangential velocity in Figures 6 and 7 consistent with the right-handed reference system of Figure 1. The abscissa in Figure 7 is the radial distance from the inner cylinder surface to the outer cylinder surface, normalised by the gap width, $d$, which is related to the cylindrical coordinates system of Figure 1(a) by $\left(r-R_{i}\right) / d$.

In Figures $7(\mathrm{a})$ and $7(\mathrm{~b})$, a more boundary layer-like velocity profile is obtained near the wall of the cylinders compared to the constant gradient profile typical of rotary viscometers or narrow gap journal bearings. The profiles show that the tangential velocity magnitude is greater than the radial velocity in Figures 2(b) and 3(b) over the range $0 \leq\left(r-R_{i}\right) / d \leq 0.94$ for $\Gamma=11.36$ and $0 \leq\left(r-R_{i}\right) / d \leq 0.82$ for $\Gamma=7.81$ and is the dominant component of the inplane velocity. This confirms the dominance of the clockwise motion shown by the velocity vector maps of Figure 5 .

In the outflow regions shown in Figure 7 (a) for $\Gamma=$ 11.36 , the normalised tangential velocity has a maximum magnitude at the inner rotating wall and zero magnitude at the outer stationary wall. Its magnitude decreases less rapidly near the wall of the inner cylinder compared to the velocity profiles through the centres of the clockwise vortices shown in Figure 8(a). The profiles then increase more rapidly near 


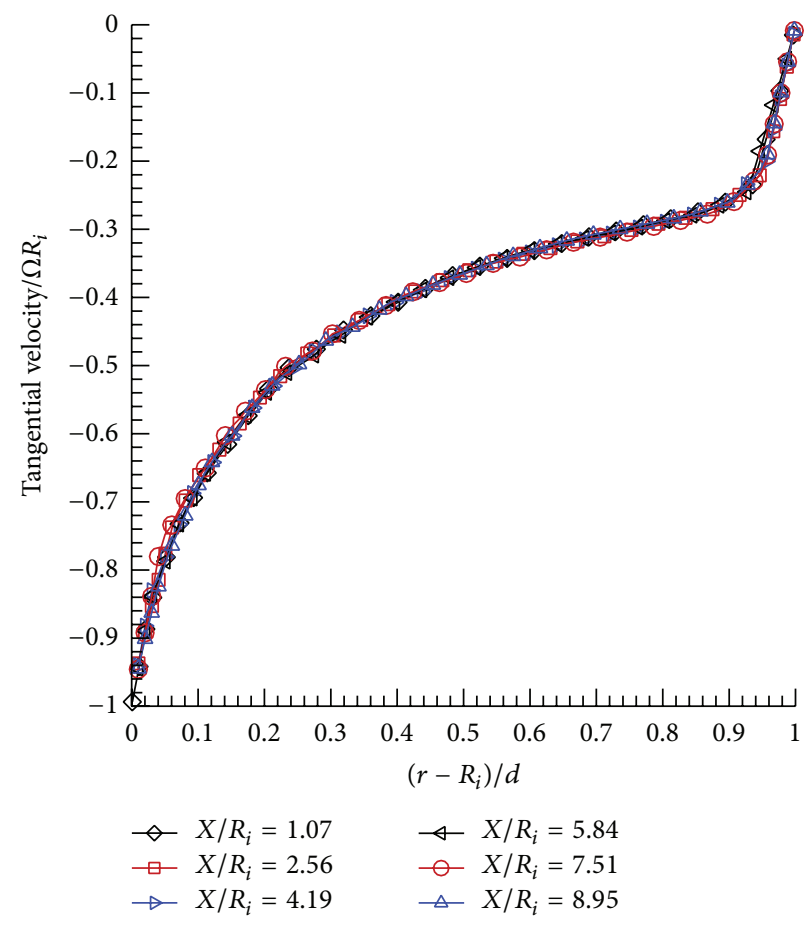

(a)

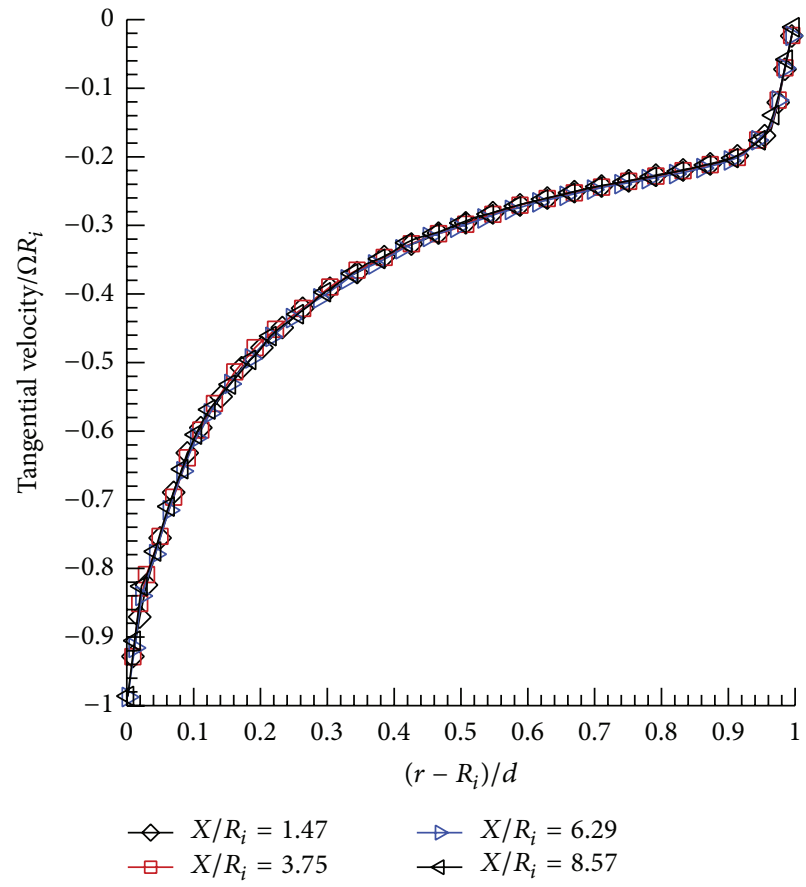

(b)

Figure 7: Normalised tangential velocity profiles at the downwell positions on selected axial planes at $\theta=-\pi / 2$ for the test cases (a) $\Gamma=11.36$ and (b) $\Gamma=7.81$.

the wall of the outer cylinder. The region with the lower tangential velocity magnitude decay rate is $0 \leq\left(r-R_{i}\right) / d \leq$ 0.16 , which corresponds to the tangential velocity range $-1.0 \Omega R_{i} \leq u_{\theta} \leq-0.63 \Omega R_{i}$. The region with rapid decay rate is $0.16 \leq\left(r-R_{i}\right) / d \leq 1.0$, which corresponds to the tangential velocity range $-0.63 \Omega R_{i} \leq u_{\theta} \leq 0$. The normalised tangential velocity magnitude in Figure $7(\mathrm{~b})$ for the test case $\Gamma=7.81$ exhibits a similar trend as in Figure 7 (a) for $\Gamma=11.36$.

The profiles through the centres of the clockwise vortices shown in Figures 8(a) and 8(b), respectively, for the test cases $\Gamma=11.36$ and $\Gamma=7.81$, show the same trend. The profiles show three distinctive regions where the profiles change. The tangential velocity magnitude near the wall of the inner cylinder decreases rapidly up to the radial position $\left(r-R_{i}\right) / d \approx 0.2$, which corresponds to a negative tangential velocity of $-0.3 \Omega R_{i}$. The tangential velocity magnitude then stabilises in the central region, over the range $0.2 \leq(r-$ $\left.R_{i}\right) / d \leq 0.90$, and then decreases rapidly over the region $0.9 \leq\left(r-R_{i}\right) / d \leq 1.0$ where it reaches zero at the wall of the outer cylinder as a result of the no-slip boundary condition.

The profiles through the centres of the anticlockwise vortices for the test cases $\Gamma=11.36$ and $\Gamma=7.81$ have a trend similar to that of Figures 8(a) and 8(b).

Figures 9(a) and 9(b) show, respectively, the tangential velocity at the inflow regions for the test cases $\Gamma=11.36$ and $\Gamma=7.81$. The tangential velocity magnitude decreases monotonically in two regions. The first region is near the inner cylinder at $0 \leq\left(r-R_{i}\right) / d \leq 0.18$, which corresponds to the tangential velocity range $-1.0 \Omega R_{i} \leq u_{\theta} \leq-0.24 \Omega R_{i}$.
The second region is $0.18 \leq\left(r-R_{i}\right) / d \leq 1.0$, which corresponds to the tangential velocity range $-0.24 \Omega R_{i} \leq u_{\theta} \leq 0$.

From the radial profiles of the in-plane normalised tangential velocity in Figures $7-9$, the tangential velocity magnitude rapidly decays from the inner rotating cylinder at all axial locations. The decay rate for the tangential velocity magnitude is more modest in the outflow regions, over the range $0 \leq\left(r-R_{i}\right) / d \leq 0.18$, compared to the decay rate over the inflow regions. It is worth noting that the tangential velocity profiles at all axial locations are different from the ones in a journal bearing where the velocity profiles are linear. A similar nonlinear behaviour of the tangential velocity profile in counterrotating cylinders was reported by Vaezi et al. [23]. In addition, the axial variation in the tangential velocity at the downwell and upwell regions is likely to lead to a significant axial variation in pressure distribution, based on simple radial equilibrium arguments in the rotating flow. This hypothesis is currently under investigation.

\section{Conclusions}

An insight into the three-dimensional dynamics of the flow in the wide gap between a stationary outer cylinder and a rotating coaxial inner cylinder was obtained by computational fluid dynamics. The wide gap configuration has received comparatively less attention than narrow gap geometries, which have been studied extensively in the literature due to their relevance to journal bearings. This study therefore fills an important gap in the literature on Taylor-Couette flows. 


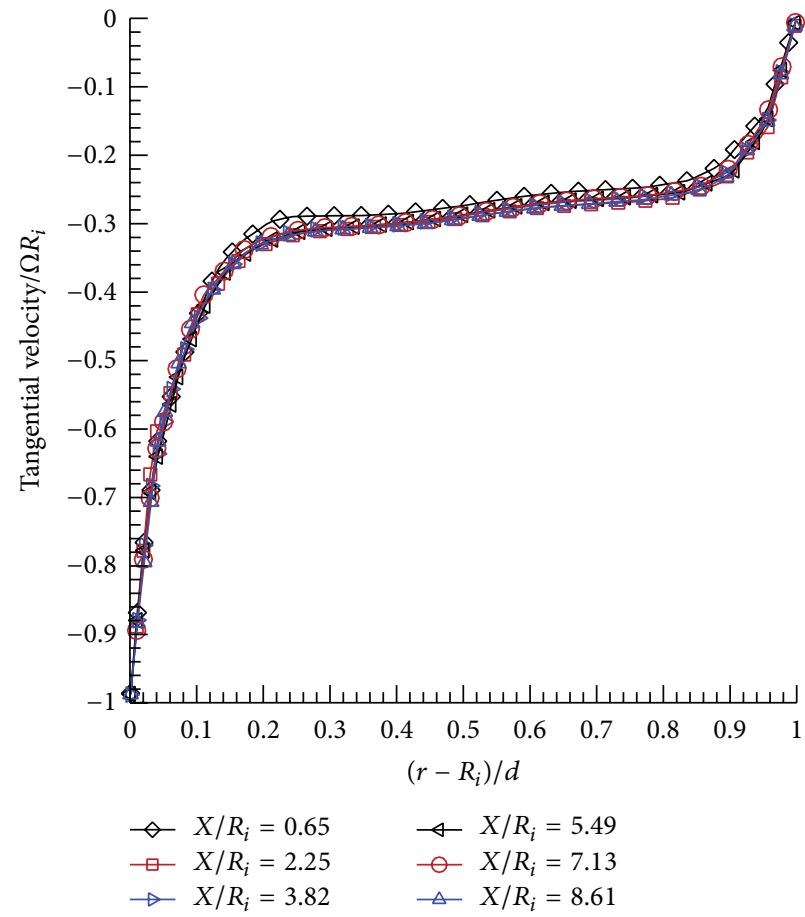

(a)

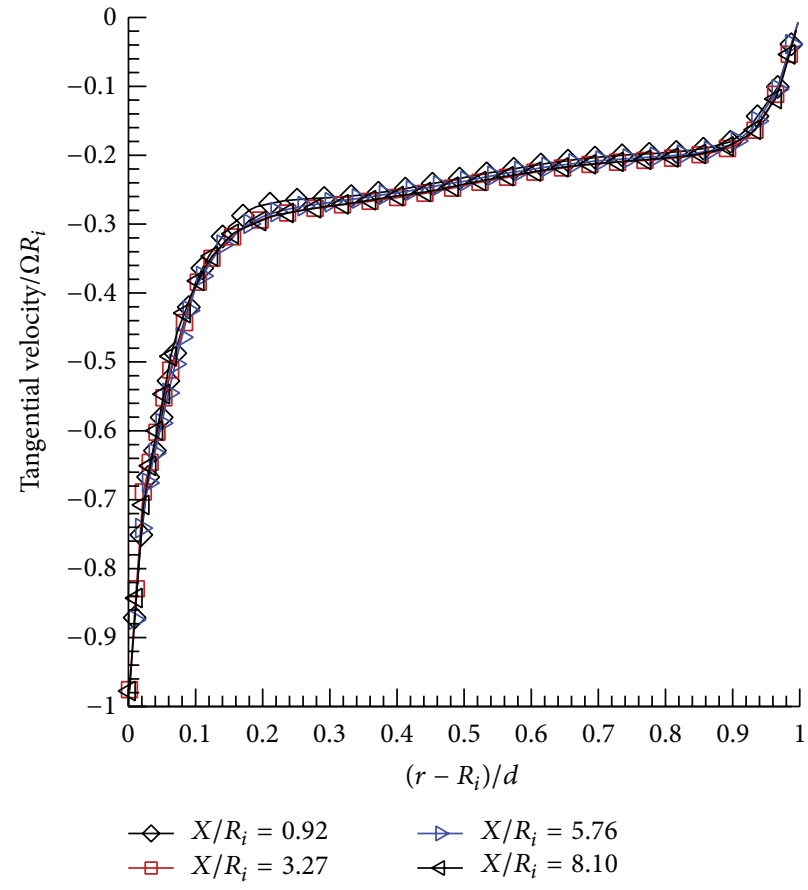

(b)

FIGURE 8: Normalised tangential velocity profiles through the centres of the clockwise vortices on selected axial planes at $\theta=-\pi / 2$ for the test cases (a) $\Gamma=11.36$ and (b) $\Gamma=7.81$.

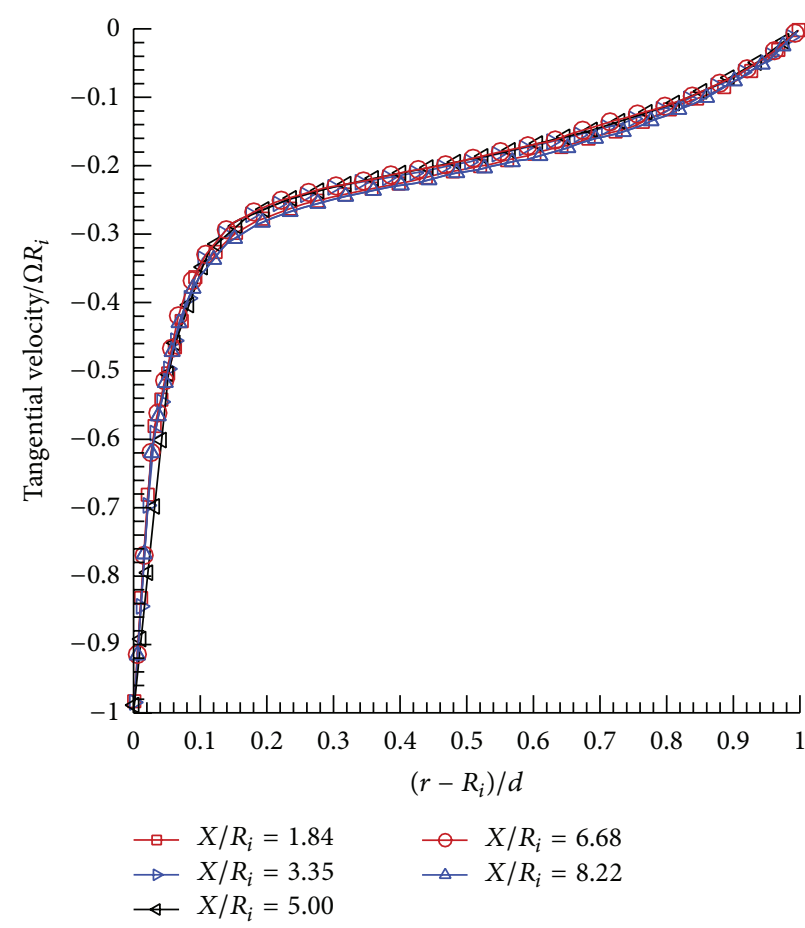

(a)

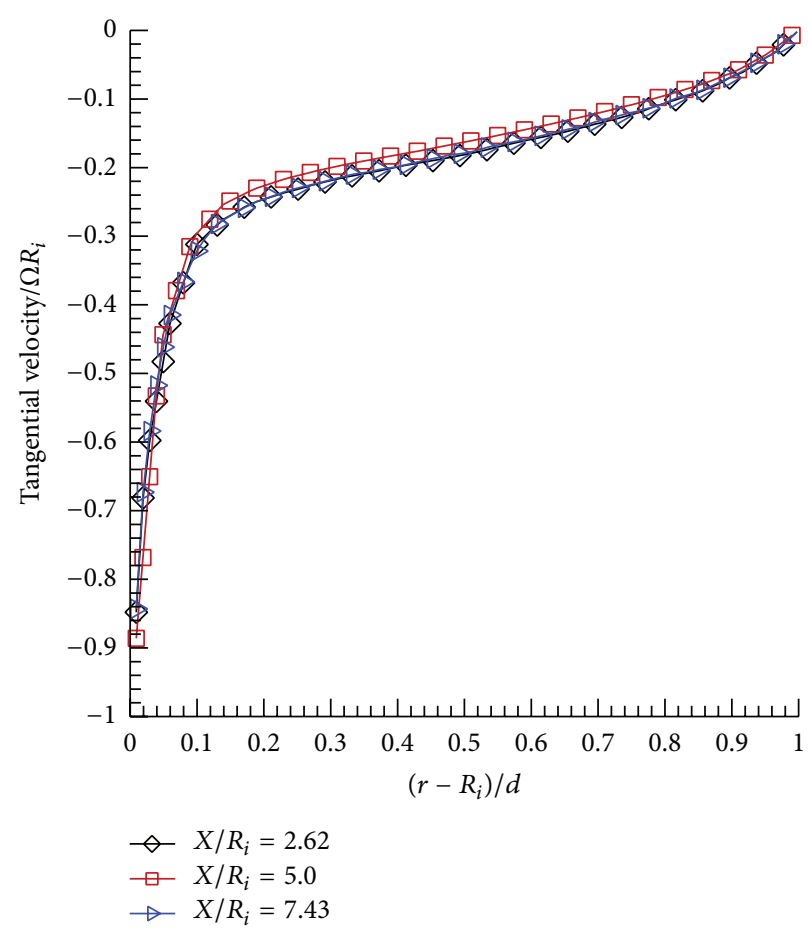

(b)

FIGURE 9: Normalised tangential velocity profiles at the upwell positions on the selected planes at $\theta=-\pi / 2$ for the test cases (a) $\Gamma=11.36$ and (b) $\Gamma=7.81$. 
Axial variations in all three velocity components document a flow that departs significantly from a two-dimensional constant gradient radial velocity distribution that may be used for representing lightly loaded roller bearing flows. The axial variation of the tangential velocity gradient induces an axially varying shear stress, resulting in local bands or rings of enhanced work input to the working fluid. This is likely to cause unwanted surface hot-spotting, arranged in the form of bands, in high torque applications.

At the axial end-wall boundaries of rotating machinery, the radial inflow is likely to promote the transport of debris to the junction between the end-collar and the rotating shaft, leading to the build-up of fouling in the shaft seal.

Whereas the current study is limited to discussing the flow dynamics, it has produced a computational fluid dynamics model that enables exploring the force and pressure distribution in the annular gap between the two cylinders. The variation in the tangential velocity along the entire annulus is likely to lead to a significant axial variation in pressure distribution, based on simple radial equilibrium arguments in the rotating flow. This hypothesis motivates a separate study dedicated to this important aspect of the Taylor-Couette system at wide gap (large clearance) that is currently underway. In addition, this study can be extended to an investigation into the effects of a non-zero axial flow rate and of a radial shaft load on the flow, towards building a higher fidelity model of a bearing chamber.

\section{Conflict of Interests}

The authors declare that there is no conflict of interests regarding the publication of this paper.

\section{Acknowledgment}

Graphical rendering software licenses were originally acquired with EPSRC support on Grant GR/N23745/01.

\section{References}

[1] H. Z. Hassan, M. H. Gobran, and A. Abd El-Azim, "Simulation of a transonic axial flow fan of a high bypass ratio turbofan engine during flight conditions," International Review of Aerospace Engineering, vol. 7, no. 1, pp. 17-24, 2014.

[2] M. Aziz, F. Owis, and M. Abdelrahman, "Preliminary design of a transonic fan for a low by-pass turbofan engine," International Review of Aerospace Engineering, vol. 6, no. 2, pp. 114-127, 2013.

[3] A. Mallock, "Determination of the viscosity of water," Proceedings of the Royal Society of London, vol. 45, pp. 126-132, 1888.

[4] A. Mallock, "Experiments on fluid viscosity," Philosophical Transactions of the Royal Society of London Series A: Mathematical, Physical and Engineering Sciences, vol. 187, pp. 41-56, 1896.

[5] M. Couette, "Études sur le frottement des liquids," Annales de Chimie et de Physique, vol. 6, pp. 433-510, 1890.

[6] G. I. Taylor, "Stability of a viscous liquid contained between two rotating cylinders," Philosophical Transactions of the Royal Society of London A, vol. 223, no. 605-615, pp. 289-343, 1923.

[7] D. Coles, "Transition in circular Couette flow," Journal of Fluid Mechanics, vol. 21, no. 3, pp. 385-425, 1965.
[8] C. B. Liao, S. J. Jane, and D. L. Young, "Numerical simulation of three-dimensional Couette-Taylor flows," International Journal for Numerical Methods in Fluids, vol. 29, no. 7, pp. 827-847, 1999.

[9] C. D. Andereck, S. S. Liu, and H. L. Swinney, "Flow regimes in a circular Couette system with independently rotating cylinders," Journal of Fluid Mechanics, vol. 164, pp. 155-183, 1986.

[10] O. Czarny, E. Serre, P. Bontoux, and R. M. Lueptow, "Spiral and wavy vortex flows in short counter-rotating Taylor-Couette cells," Theoretical and Computational Fluid Dynamics, vol. 16, no. 1, pp. 5-15, 2002.

[11] D. S. Adebayo and A. Rona, "The persistence of vortex structures between rotating cylinders in the $10^{6}$ Taylor number range," International Review of Aerospace Engineering, vol. 8, no. 1, pp. 16-25, 2015.

[12] D. S. Adebayo and A. Rona, "PIV study of the flow across the meridional plane of rotating cylinders with wide gap," International Review of Aerospace Engineering, vol. 8, no. 1, pp. 26-34, 2015.

[13] ANSYS Fluent, Fluent 12.0 User's Manual Guide, Fluent Incorporated, Lebanon, NH, USA, 2009.

[14] E. L. Koschmieder, “Turbulent Taylor vortex flow," Journal of Fluid Mechanics, vol. 93, no. 3, pp. 515-527, 1979.

[15] S. S. Deshmukh, S. Vedantam, J. B. Joshi, and S. B. Koganti, "Computational flow modeling and visualization in the annular region of annular centrifugal extractor," Industrial and Engineering Chemistry Research, vol. 46, no. 25, pp. 8343-8354, 2007.

[16] H. K. Versteeg and W. Malalasekera, An Introduction to Computational Fluid Dynamics: The Finite Volume Method, Pearson Prentice Hall, Essex, UK, 1995.

[17] J. D. Anderson, Computational Fluid Dynamics: The Basics with Applications, McGraw-Hill, New York, NY, USA, 1995.

[18] A. J. Chorin, "Numerical solution of the Navier-Stokes equations," Mathematics of Computation, vol. 22, pp. 745-762, 1968.

[19] T.-H. Shih, W. W. Liou, A. Shabbir, Z. Yang, and J. Zhu, "A new k-epsilon eddy-viscosity model for high Reynolds number turbulent flows-model development and validation," Computers \& Fluids, vol. 24, no. 3, pp. 227-238, 1995.

[20] B. E. Launder, G. J. Reece, and W. Rodi, "Progress in the development of a Reynolds-stress turbulent closure," Journal of Fluid Mechanics, vol. 68, no. 3, pp. 537-566, 1975.

[21] F. Wendt, "Turbulente strömungen zwischen zwei rotierenden konaxialen zylindern," Ingenieur-Archiv, vol. 4, no. 6, pp. 577595, 1933.

[22] D. P. Lathrop, J. Fineberg, and H. L. Swinney, "Transition to shear-driven turbulence in Couette-Taylor flow," Physical Review A, vol. 46, no. 10, pp. 6390-6405, 1992.

[23] V. Vaezi, E. S. Oh, and R. C. Aldredge, "High-intensity turbulence measurements in a Taylor-Couette flow reactor," Experimental Thermal and Fluid Science, vol. 15, no. 4, pp. 424431, 1997. 


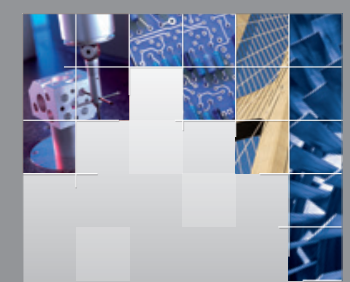

\section{Enfincering}
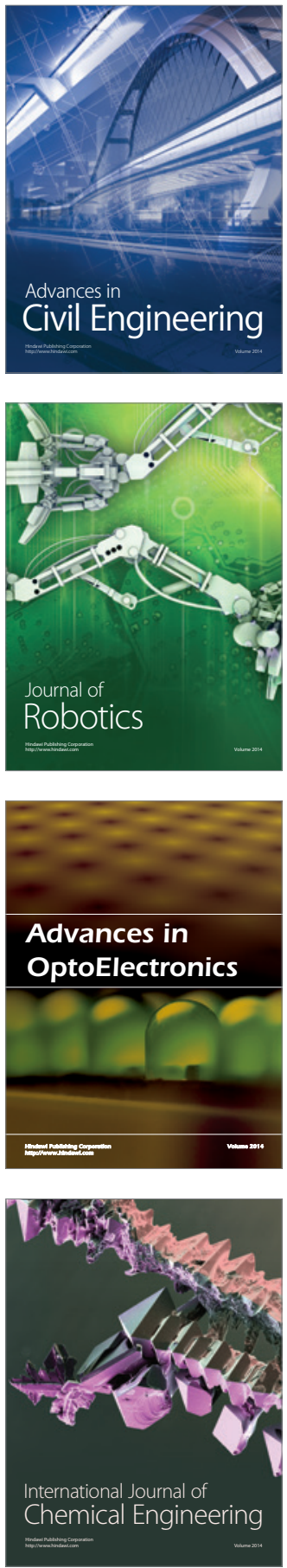

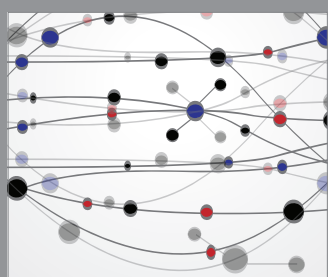

The Scientific World Journal

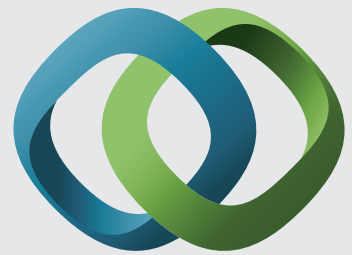

\section{Hindawi}

Submit your manuscripts at

http://www.hindawi.com
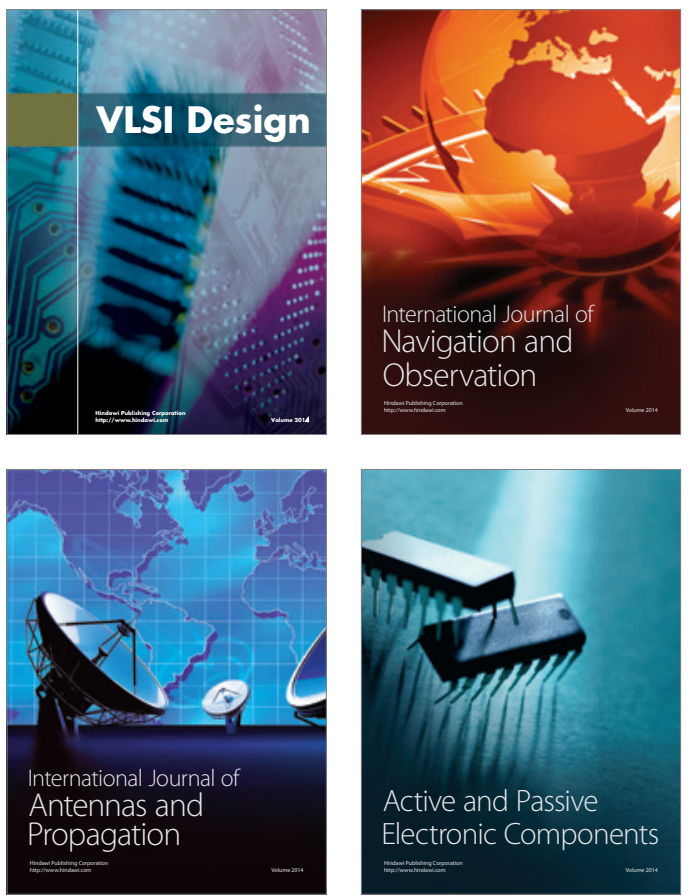
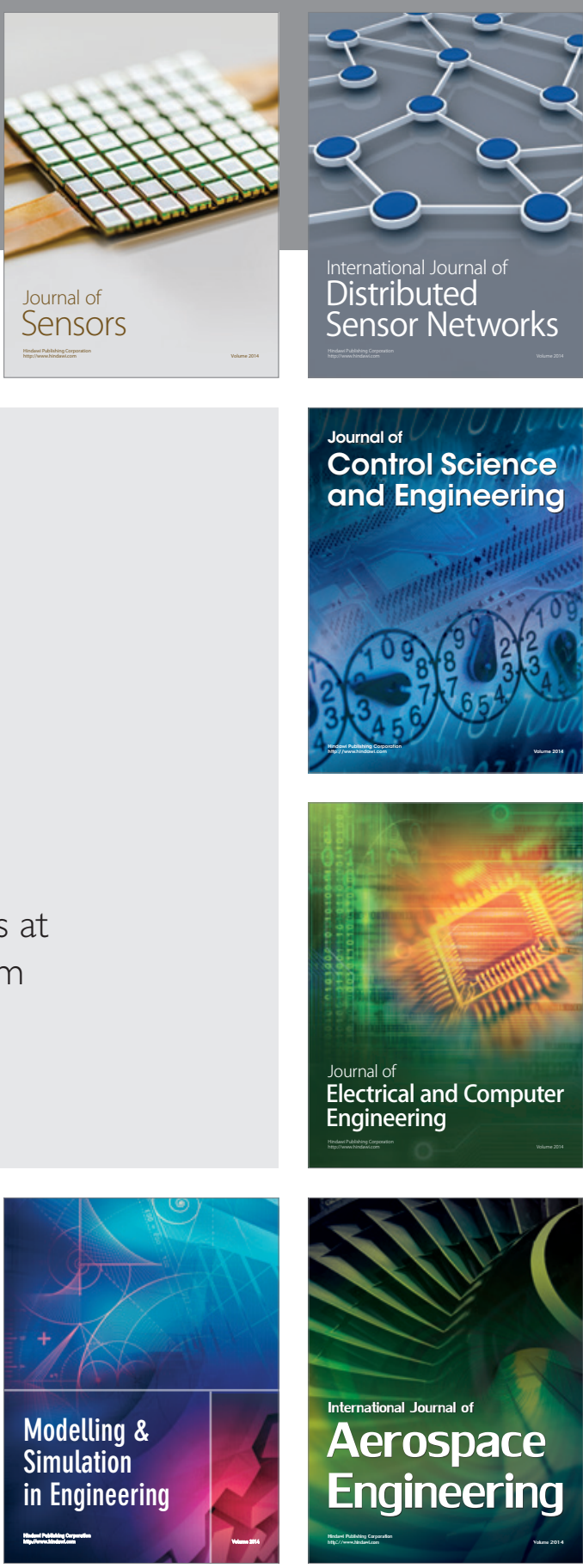

International Journal of

Distributed

Sensor Networks

Journal of

Control Science

and Engineering
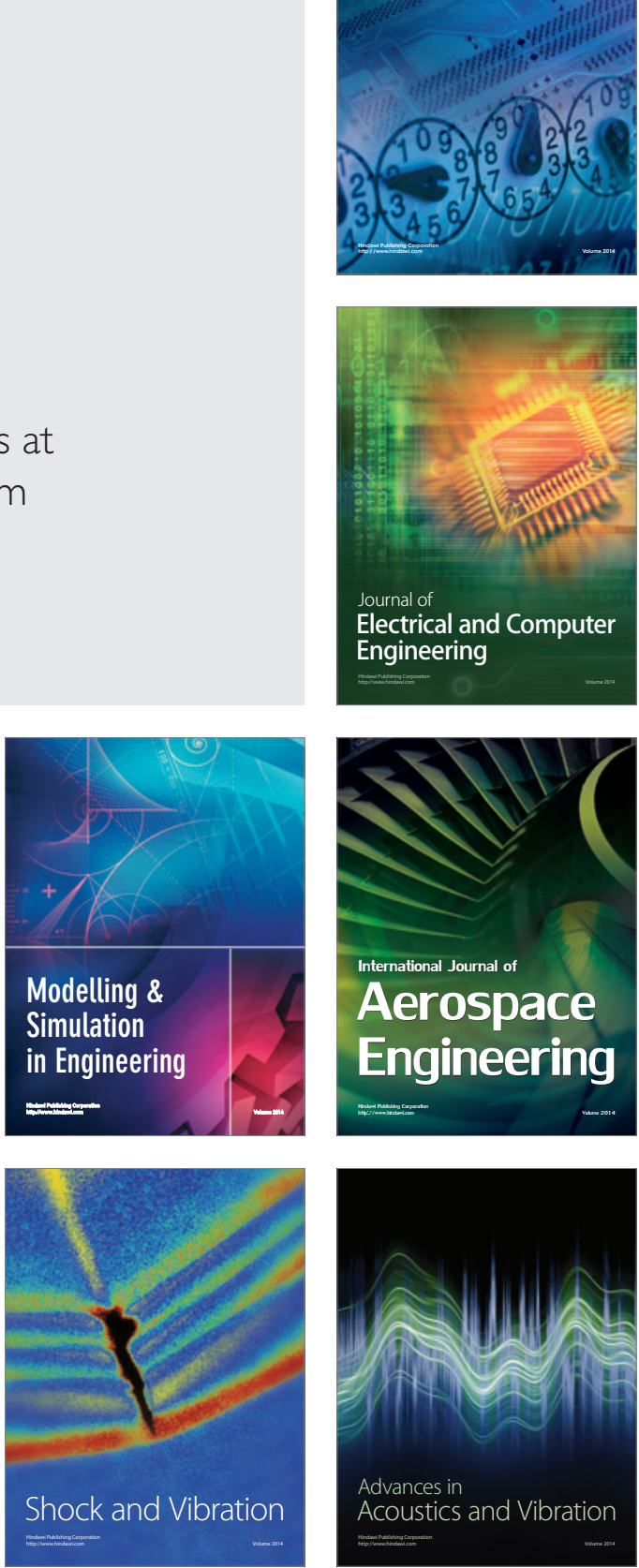